\title{
Experiments on low-Reynolds-number turbulent flow through a square duct
}

\author{
Bayode E. Owolabi ${ }^{1}$, Robert J. Poole ${ }^{1}$ and David J. C. Dennis ${ }^{1, \dagger}$ \\ ${ }^{1}$ School of Engineering, University of Liverpool, Liverpool L69 3GH, UK \\ (Received 18 August 2015; revised 28 February 2016; accepted 1 May 2016; \\ first published online 3 June 2016)
}

\begin{abstract}
Previous experimental studies on turbulent square duct flow have focused mainly on high Reynolds numbers for which a turbulence-induced eight-vortex secondary flow pattern exists in the cross-sectional plane. More recently, direct numerical simulations (DNS) have revealed that the flow field at Reynolds numbers close to transition can be very different; the flow in this 'marginally turbulent' regime alternating between two states characterised by four vortices. In this study, we experimentally investigate the onset criteria for transition to turbulence in square ducts. In so doing, we highlight the potential importance of Coriolis effects on this process for low-Ekman-number flows. We also present experimental data on the mean flow properties and turbulence statistics in both marginally and fully turbulent flow at relatively low Reynolds numbers using laser Doppler velocimetry. Results for both flow categories show good agreement with DNS. The switching of the flow field between two flow states at marginally turbulent Reynolds numbers is confirmed by bimodal probability density functions of streamwise velocity at certain distances from the wall as well as joint probability density functions of streamwise and wall normal velocities which feature two peaks highlighting the two states.
\end{abstract}

Key words: instability, transition to turbulence, turbulent flows

\section{Introduction}

Following the findings of Nikuradse (1926) on the existence of secondary currents in square duct turbulent flow, further experimental studies (Hoagland 1960; Brundrett \& Baines 1964; Launder \& Ying 1972; Melling \& Whitelaw 1976) have been conducted to obtain a better understanding of the phenomenon, which came to be known as 'Prandtl's secondary flow of the second kind'. These studies, which focused mainly on turbulent flow at relatively high Reynolds numbers $(R e>35000$; $R e=U_{b} h / v$ where $U_{b}, h$ and $v$ represent the bulk velocity, duct half height and kinematic viscosity, respectively) have shown that secondary flow, though weak in magnitude, has a considerable impact on the primary flow. Developments in computational fluid dynamics have made possible the direct numerical simulation 
(DNS) of such flows to complement experimental results and provide more insight into the turbulence structure. However computational restrictions currently limit DNS to low Reynolds numbers $(R e<5000)$, hence comparisons between experiments and simulations have mostly been qualitative. At such Reynolds numbers, the turbulent flow field has been found to behave differently from what is observed experimentally at much higher Reynolds numbers.

One of the earliest DNS of square duct turbulent flow was conducted by Gavrilakis (1992) at $R e=2205$. His results show good qualitative agreement with experimental findings with regards to bulging of the contour of mean streamwise velocity towards the duct corners and an eight-vortex secondary flow pattern. However, the wall shear stress $\left(\tau_{w}\right)$ was found to be non-uniformly distributed across the duct due to the secondary flow field. Specifically, three peaks were observed on each wall; one at the midpoint and the other two close to the corners. Another key finding from the simulation, which is a consequence of these wall shear stress gradients, was that the streamwise velocity profile along the wall bisector exhibited an overshoot from the well-known logarithmic law $\left(u^{+}=2.5 \ln y^{+}+5.5\right)$ in the overlap layer when normalised by the average (across the duct perimeter) rather than local friction velocity, $u_{\tau}=\sqrt{\tau_{w} / \rho}$, where $\rho$ is the density. Other researchers who have conducted DNS of square duct turbulent flow and obtained similar results include Huser \& Biringen (1993) and Joung, Choi \& Choi (2007).

A pertinent research question which, until recently, has received little attention in the literature is the determination of the mechanism for transition to turbulence in square duct flow and the flow field characteristics at the edge of turbulence. Like pipe flow, square duct flow is linearly stable at all Reynolds numbers (Tatsumi \& Yoshimura 1990). However, occurrence of exact coherent structures such as travelling waves is believed to signal the beginning of chaos. Travelling wave solutions have been discovered by Wedin, Bottaro \& Nagata (2009), Okino et al. (2010) and Uhlmann, Kawahara \& Pinelli (2010) at $R e=598.2,332$ and 471 respectively.

Numerical simulations reveal that the limiting value of Reynolds number for the onset of self-sustaining turbulence in periodic square-duct flow ranges between $R e=865\left(R e_{\tau}=65\right)$ and $R e=1077\left(R e_{\tau}=80\right)$, where $R e_{\tau}$ is the Reynolds number based on friction velocity and duct half-height (Biau \& Bottaro 2009; Uhlmann et al. 2007). The DNS results of Uhlmann et al. (2007) are particularly fascinating. So-called marginally turbulent flow was found to be characterised by two flow states each of which contained a four-vortex secondary flow field rather than the conventional pattern of eight vortices. The vortex pairs, which were associated with a pair of opposite walls, alternated between two different orientations, the eight-vortex secondary flow pattern being a composite of these two as reproduced in figure 1. This switching of the flow field between two states in the marginally turbulent regime has not previously been observed in experiments. This different secondary flow pattern was attributed to buffer layer coherent structures (streaks), whose preferential positioning over the duct walls matched those of the vortices.

In two follow-up papers, changes to the flow field as fully turbulent flow is approached were investigated (Sekimoto et al. 2009; Pinelli et al. 2010). The distribution of wall shear stress was found to be correlated to the number and positioning of buffer layer streaks (which increased with Reynolds number), the high-speed streaks always being preferentially located in the corner region, leading to local maxima in the wall shear stress distribution. On the other hand, low-speed streaks were associated with the occurrence of local minima. This explains the two peaked wall shear stress distribution observed in DNS of marginally turbulent flows. Again, there is lack of experimental data for comparison. 
(a)

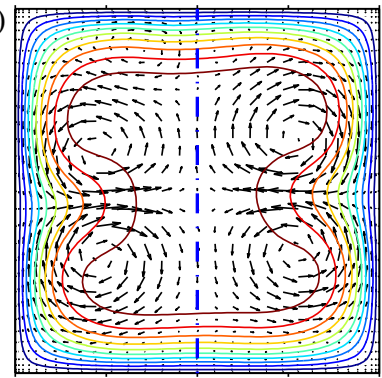

(b)

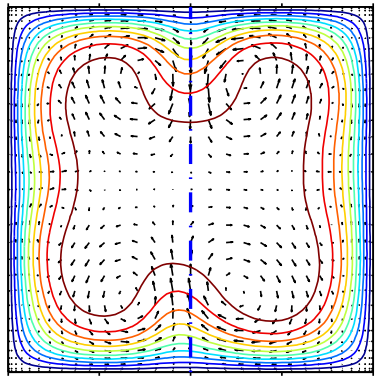

(c)

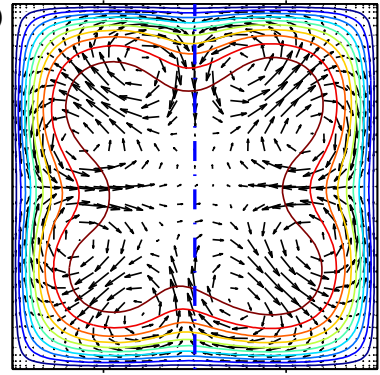

FiguRE 1. (Colour online) The two flow states of marginally turbulent flow $(a, b)$ and the conventional eight-vortex pattern $(c)$. Adapted from figure 3 of Uhlmann et al. (2007). Contour lines show the primary mean flow $\langle U\rangle$ and vectors show the secondary mean flow $\langle V\rangle,\langle W\rangle$ for $R e=1205$ : (a) averaging interval $771 h / U_{b} ;(b)$ a different interval with length $482 h / U_{b} ;(c)$ long-time integration including both previous intervals $\left(1639 h / U_{b}\right)$. The wall bisector is indicated by dashed blue lines.

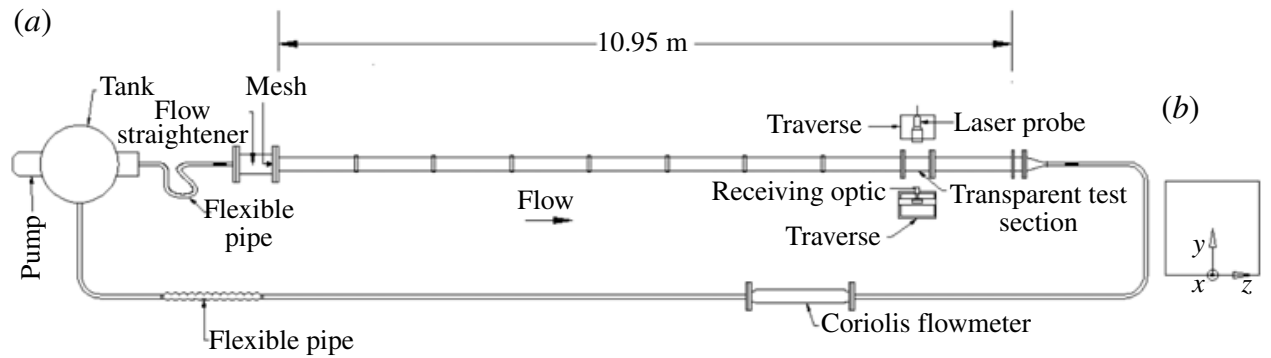

FIgURE 2. Experimental set-up. (a) Schematic of the flow loop (not to scale). (b) Axes system employed; $y$ is the wall-normal direction measured from the nearest wall. The streamwise/axial direction, $x$, is into the page.

It is the aim of this study, therefore, to provide experimental data on turbulent flow in a square duct at relatively low Reynolds numbers in order to investigate these marginally turbulent states and provide validation data for DNS.

\section{Experimental set-up}

The experimental rig used for this study is a modified version of that employed by Escudier \& Smith (2001). The working section (as shown in figure 2a) consists of eight square duct modules made of stainless steel, each of length $1.2 \mathrm{~m}$ and cross-sectional dimensions of $80 \mathrm{~mm} \times 80 \mathrm{~mm}(2 h \times 2 h)$, followed by a transparent section $150 \mathrm{~mm}$ in length, constructed from Perspex and another stainless steel module, $1.2 \mathrm{~m}$ long, bringing the total length to $10.95 \mathrm{~m}$. With this arrangement, there is a distance of about $240 \mathrm{~h}$ before the transparent section, where laser Doppler velocimetry (LDV) measurements were taken, which is enough for both fully developed laminar and turbulent flow at the Reynolds numbers studied.

A progressive cavity Mono pump fed by the working fluid stored in a stainless steel tank drives the flow. The pump and tank are connected to the working section using a flexible corrugated pipe, $5 \mathrm{~m}$ in length, which serves as a pulsation damper. This is followed by a short section that provides a gradual transition from a circular to square 
cross-section, which includes a honeycomb flow straightener and an additional fine mesh to remove any disturbances from the pump and inlet. The fluid is recirculated through the rig via a return loop consisting of polyvinyl chloride pipes. The mass flow rate as well as fluid temperature and density were measured using an Endress and Hauser Promass I Coriolis flow meter installed in the return loop.

Velocity measurements were taken using a two-dimensional (2D) Dantec fibreflow LDV system operated in forward scatter mode. This system consisted of a $60 \times 10$ probe, $55 \times 12$ beam expander and an argon-ion laser source which supplied lights of wavelengths $515.5 \mathrm{~nm}$ (green) and $488 \mathrm{~nm}$ (blue) for resolving the velocity components in the streamwise and wall-normal directions (respectively $x$ and $y$ as defined in figure $2 b$ ). The front lens of the laser probe had a focal length of $160 \mathrm{~mm}$ and a beam separation distance of $51.5 \mathrm{~mm}$, resulting in a measuring volume of diameter $24 \mu \mathrm{m}$ and length $150 \mu \mathrm{m}$ in air. With this configuration, the typical data rates were around $100 \mathrm{~Hz}$ in non-coincidence mode and $20 \mathrm{~Hz}$ in coincidence mode. At each wall-normal location, data was collected for typically $30 \mathrm{~min}$ in the laminar and fully turbulent regimes and for up to $1 \mathrm{~h}$ in the marginally turbulent state. The region below $8 \mathrm{~mm}$ from the duct wall was not accessible to the laser beams in the wall-normal plane; hence, data on wall-normal velocity components in that area could not be collected. Signal processing was carried out using a Dantec Burst Spectrum analyser (model F50) while data acquisition and processing was done using the Dantec BSA flow software (version 2.12.00.15). The fluids used were either water $\left(v \approx 10^{-6} \mathrm{~m}^{2} \mathrm{~s}^{-1}\right)$ or $50 \%$ glycerol/water solution $\left(v \approx 6 \times 10^{-6} \mathrm{~m}^{2} \mathrm{~s}^{-1}\right)$ depending on the Reynolds number of interest. The local wall shear stress was determined by taking measurements of the velocity profile in the viscous sublayer, obtaining the gradient of the resulting linear plot and applying the expression: $\tau_{w}=\mu(\mathrm{d}\langle U\rangle / \mathrm{d} y)$, where $\langle U\rangle$ is the mean streamwise velocity. The local friction velocity $\left(u_{\tau}\right)$ was subsequently estimated from this value of wall shear stress. Unlike in hot-wire anemometry, this technique is free from the so-called wall effect since LDV is non-intrusive. The very small measuring volume minimises any potential errors due to velocity gradient broadening. A detailed discussion on wall shear stress determination using the near-wall velocity gradient method is given by Hutchins \& Choi (2002).

\section{Transition to turbulence}

\subsection{Experimental studies}

It is well known that a turbulent flow field can be split into a mean and fluctuating component. Steady laminar flow is characterised by no fluctuations, hence an increase in intensity of the fluctuating velocity component is a good measure for detecting the onset of transition to turbulence in ducts. Figure $3(a, b)$ shows the variation of mean streamwise velocities, $\langle U\rangle$, normalised by the bulk velocity, $U_{b}$, and the root mean square of the fluctuations, $u_{r m s}$, with Reynolds number at the duct centreline $(y / h=1.0)$. The data have been obtained using water.

In figure 3(a), a drop off in the values of $\langle U\rangle / U_{b}$ from the laminar flow analytical solution (White 2006, p. 113) can be observed for $R e<1000$. This deviation from the analytical solution is surprising as the velocity fluctuations remain very low (about $2 \%$ ), indicating that the flow is laminar (see figure $3(b)$; the fluctuations, which ideally should be zero, can be attributed to the measurement noise of the LDV system). We believe this deviation of the observed $\langle U\rangle / U_{b}$ from the analytical solution can be 

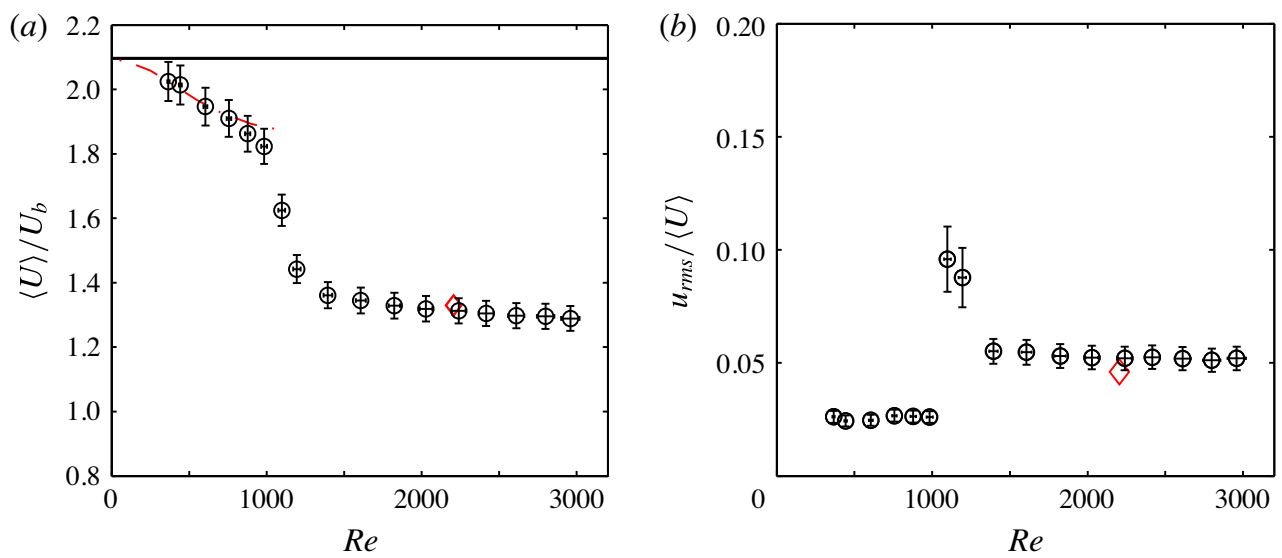

FIgURE 3. (Colour online) Onset criteria for square duct turbulent flow $(E k \approx 1, y / h=1)$. (a) Variation of $\langle U\rangle / U_{b}$ with Reynolds number. (b) Variation of $u_{r m s} /\langle U\rangle$ with Reynolds number. - laminar flow analytical solution at $y / h=1 ; \diamond($ red), DNS of Gavrilakis (1992); $-\cdot \cdot \cdot-$ (red), numerical simulation of laminar flow at $E k=1$.

ascribed to Coriolis effects due to the Earth's rotation as has been observed previously in pipe flows by Draad \& Nieuwstadt (1998). They showed that the effect of rotation on a fully developed laminar flow can be estimated using the Ekman number, a ratio of viscous to Coriolis forces:

$$
E k=\frac{v}{2 \Omega D^{2} \sin \alpha},
$$

where $\Omega$ is the angular velocity of the Earth $\left(7.272 \times 10^{-5} \mathrm{~s}^{-1}\right), D$ is the duct width $(0.08 \mathrm{~m})$ and $\alpha$ the angle between the duct axis and the Earth's rotation axis $\left(\approx 69^{\circ}\right)$. In our case, we obtain $E k \approx 1$ for water, hence Coriolis effects cannot be ignored in the laminar regime for this fluid. The Coriolis force brings about a distortion in the laminar flow velocity profile, which should normally be parabolic and symmetrical about the wall bisector, by introducing acceleration components in the wall-normal directions.

Transition can be said to take place at $R e \approx 1050$ as evidenced by a significant drop in the value of $\langle U\rangle / U_{b}$ from those of laminar flow. At this point, turbulence bursts are introduced into the laminar flow, hence the apparently large fluctuation levels recorded in figure $3(b)$. As the Reynolds number is increased, $u_{r m s} /\langle U\rangle$ settles down to about $5 \%$, the turbulence having become self-sustaining, and $\langle U\rangle / U_{b}$ gradually approaches the fully turbulent value obtained from the DNS of Gavrilakis (1992). In turbulent flow, inertial forces dominate and Coriolis effect becomes negligible.

To reduce the effect of Coriolis force in the laminar regime, we make use of a more viscous liquid $(50 \%$ glycerol/water solution) resulting in $E k \approx 7$. This fluid is employed for all subsequent experiments. Figure $4(a, b)$ show the variation of $\langle U\rangle / U_{b}$ and $u_{r m s} /\langle U\rangle$ with Reynolds number. The experimental data for $\langle U\rangle / U_{b}$ at $y / h=1$ can be observed to agree well with the laminar flow analytical solution until $R e \approx 800$ where there is a drop off once again due to the Coriolis effect. This is in agreement with the findings of Escudier et al. (2005) who obtained laminar velocity profiles in pipe flow which were slightly asymmetric for $R e$ as low as 540 for $E k \approx 5$. Transition to turbulence can be observed to take place at $R e \approx 1250$ as indicated by an increase 

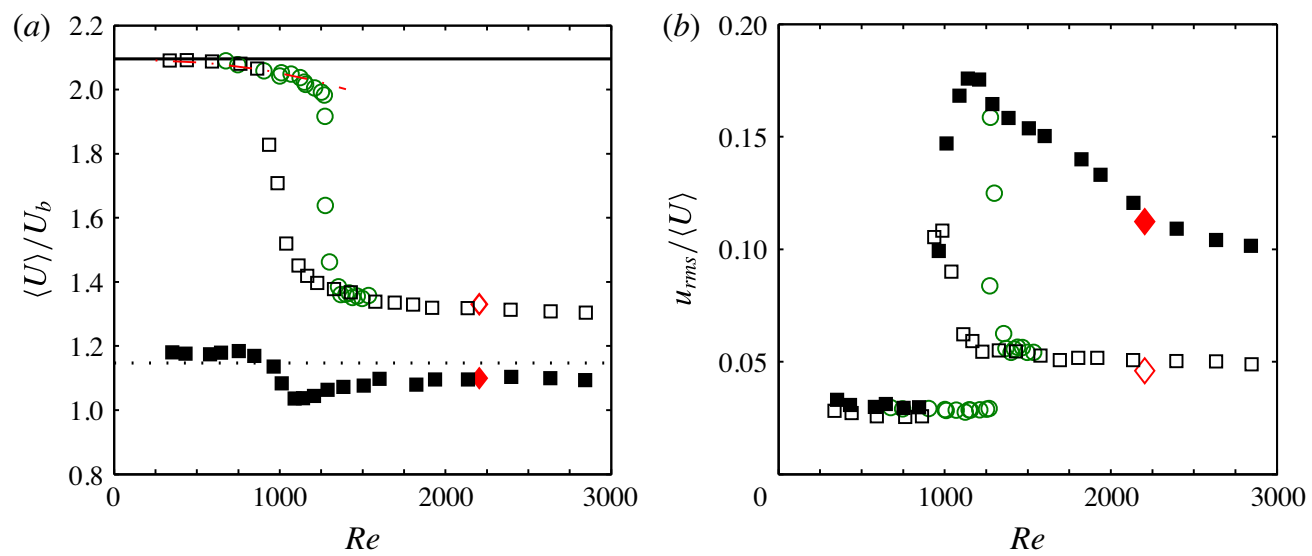

FIGURE 4. (Colour online) Onset criteria for square duct turbulent flow $(E k \approx 7)$. (a) Variation of $\langle U\rangle / U_{b}$ with Reynolds number. (b) Variation of $u_{r m s} /\langle U\rangle$ with Reynolds number. Open symbols, $y / h=1$; closed symbols, $y / h=0.3$; $\square$, trip rod introduced upstream; $\bigcirc$ (green), no trip rod upstream; —- laminar flow analytical solution at $y / h=1 ; \cdots \cdots$, laminar flow analytical solution at $y / h=0.3 ; \diamond($ red), DNS of Gavrilakis (1992); $-\cdot \cdot \cdot-($ red), numerical simulation of laminar flow at $E k=7$.

in velocity fluctuation levels as well as a significant drop in $\langle U\rangle / U_{b}$ from the laminar flow analytical solution. The difference in transition Reynolds number between water and $50 \%$ glycerol/water solution can be attributed to their dissimilar laminar base profiles prior to transition.

To determine the lowest Reynolds number for the onset of sustained turbulence, we introduce a trip rod upstream (about $238 h$ from the measurement section). In this instance transition can be observed to take place at $R e \approx 940$. This value of $R e$ is within the range given by Uhlmann et al. (2007) and Biau \& Bottaro (2009): $R e=1077$ and 865, respectively. It is however higher than those where previous studies have shown the emergence of travelling wave solutions, which are known to be precursors to fully developed turbulence. At $y / h=0.3$, the streamwise velocity, normalised by the bulk velocity can be observed to be roughly constant in the laminar regime. It however drops off during transition before approaching the fully turbulent value given by Gavrilakis (1992) which, coincidentally, is close to the laminar flow value. The velocity fluctuations at $y / h=0.3$ after transition are much higher than those at the duct centre and attain that of fully turbulent flow more slowly (see figure $4 b$ ). This lends credence to the idea that the important structures that govern the dynamics of transition in square duct flow are primarily located in (and around) the buffer layer.

\subsection{Numerical simulation of square duct laminar flow under the influence of Coriolis force}

Following the approach of Draad \& Nieuwstadt (1998), we carry out a numerical simulation of fully developed laminar square duct flow, including the effect of the Earth's rotation in order to confirm our hypothesis that these effects cannot be neglected. The governing Navier-Stokes equations for the flow are as follows:

$$
\frac{\mathrm{D} U_{i}}{\mathrm{D} t}=f_{i}-\frac{1}{\rho} \frac{\partial p}{\partial x_{i}}+v \frac{\partial^{2} U_{i}}{\partial x_{j}^{2}},
$$


where $U_{i}$ and $f_{i}$ denote the velocity field and Coriolis force per unit mass, respectively, and $p$ is the pressure term accounting for centrifugal forces. Considering the axis system of figure $2(b)$, and a unidirectional flow vector $[U(x, y, z), 0,0]$, The Coriolis force per unit mass is given by $\boldsymbol{f}=-2 \boldsymbol{\Omega} \times \boldsymbol{U}$, where $\boldsymbol{\Omega}$ is the Earth's angular velocity vector given by

$$
\boldsymbol{\Omega}=\Omega\left(\begin{array}{c}
\cos \alpha_{L} \cos \alpha_{N} \\
\sin \alpha_{L} \\
-\cos \alpha_{L} \sin \alpha_{N}
\end{array}\right),
$$

$\alpha_{L}$ and $\alpha_{N}$ being the latitude (53 ${ }^{\circ}$ in our case) and the angle between the direction of true north and the duct axis $\left(100^{\circ}\right)$, respectively. Hence,

$$
\left(\begin{array}{l}
f_{x} \\
f_{y} \\
f_{z}
\end{array}\right)=-2 \Omega U(x, y, z)\left(\begin{array}{c}
0 \\
-\cos \alpha_{L} \sin \alpha_{N} \\
-\sin \alpha_{L}
\end{array}\right) .
$$

We solve the Navier-Stokes equations using the commercial software, ANSYS Fluent, for a domain: $D \times D \times 110 D$ with $50 \times 50 \times 2160$ grid points (uniform in the crosssectional plane, but non-uniform in the streamwise direction such that the ratio of the largest/first to the smallest/last cell is 7.5), using a pressure-based coupled solver. This software makes use of the finite volume approach. Spatial discretisation of pressure, gradient and momentum were carried out using the least-squares cell-based, standard and second-order upwind schemes, respectively.

Numerical simulation results at $y / h=1$ for $E k=1$ and 7 are shown as dot-dashed lines in figures $3(a)$ and $4(a)$ respectively. The results show good agreement with the experimental data. The deviation of $\langle U\rangle / U_{b}$ from the laminar flow analytical solution can be observed to increase with Reynolds number. This is due to an increase in laminar velocity profile asymmetry, the level of distortion being higher for $E k=1$. Given the previous results of Draad \& Nieuwstadt (1998) and the excellent agreement between experiment and simulation here for two different Ekman numbers, we conclude that Coriolis forces can be significant in fully developed laminar square duct flow, especially for water. As a consequence, all of the data which follows is for $50 \%$ glycerol/water solution $(E k \approx 7)$.

\section{Characteristics of low-Reynolds-number turbulent flows}

\subsection{Mean streamwise velocity measurements}

Figure 5(a) shows the profile of streamwise velocity normalised by the bulk velocity, along the wall bisector, for both marginally turbulent $\left(R e=1203, R e_{\tau}=81\right)$ and fully turbulent $\left(R e=2230, R e_{\tau}=161\right)$ flow. The DNS results of Gavrilakis (1992) at a Reynolds number of $2205\left(R e_{\tau}=162\right)$ and Uhlmann et al. (2007) at a Reynolds number of $1205\left(R e_{\tau}=84\right)$ as well as the laminar flow analytical solution (White 2006) are also presented for the purpose of comparison. The flow was found to be symmetric, hence only the data from the lower half of the duct along the vertical bisector is presented, $y$ being the distance from the bottom wall as shown in figure $2(b)$.

The experimental results show good agreement with DNS. It is interesting to note that the velocity gradient at the wall at $R e=1203$ is very similar to that of laminar flow. However, beyond $y / h \approx 0.3$, the mean flow becomes markedly different from the laminar case. The values of $\langle U\rangle / U_{b}$ at the duct centre are 1.40 and 1.31 for marginally and fully turbulent flow, respectively. 

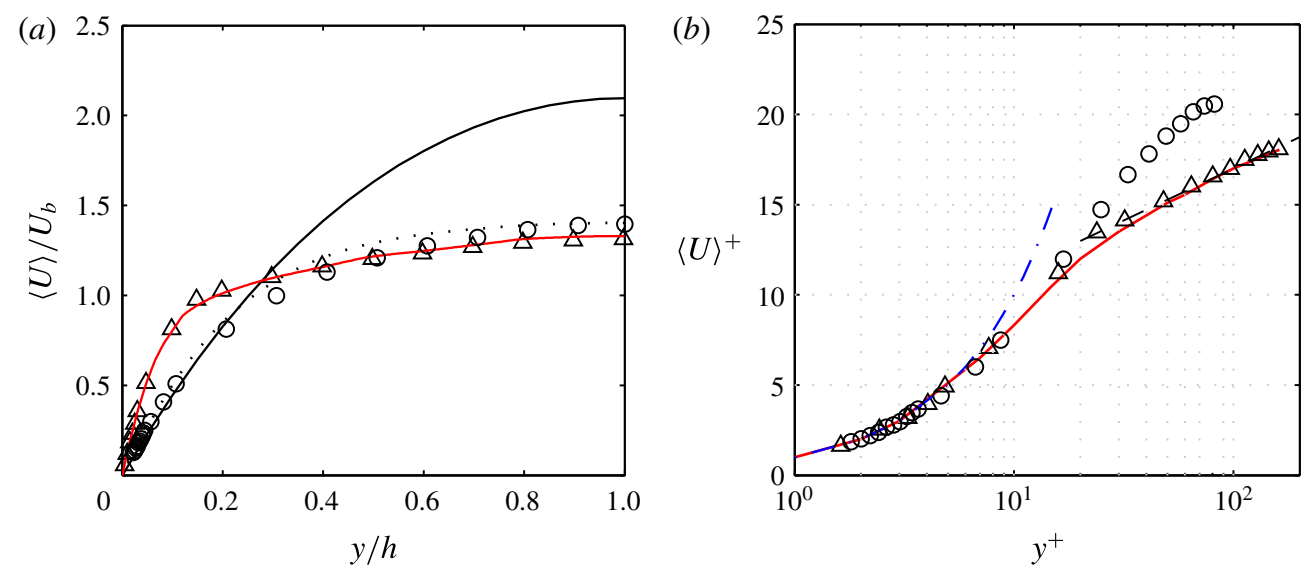

FIGURE 5. (Colour online) Axial velocity profiles along the wall bisector. (a) In outer units. (b) In wall units. $\bigcirc$, experiment at $R e=1203\left(R e_{\tau}=81\right) ; \Delta$, experiment at $R e=2230\left(R e_{\tau}=161\right) ; \cdots \cdots$, DNS of Uhlmann et al. (2007) at $R e=1205$; - (red), DNS of Gavrilakis (1992) at $R e=2205\left(R e_{\tau}=162\right)$; - laminar flow analytical solution (White 2006). ----, $u^{+}=2.5 \ln y^{+}+5.5 ;-\cdot-\cdot-$, (blue), $u^{+}=y^{+}$.

In figure $5(b)$, the same data is plotted in wall units, indicated by the superscript + , where the velocities have been normalised by local friction velocity $\left(u_{\tau}\right)$. Again, data at $R e_{\tau}=161$ show excellent agreement with the DNS of Gavrilakis (1992). A large overshoot from the logarithmic law can be observed in the data for $R e_{\tau}=81$, confirming that the wall shear stress distribution is characterised by a local minimum rather than maximum at the duct midpoint in marginally turbulent flows, as shown by Pinelli et al. (2010).

\subsection{Instantaneous velocity measurements}

The probability density functions (p.d.f.s) of instantaneous streamwise velocity, $U$, normalised by either local mean, $\langle U\rangle$ or bulk velocity, $U_{b}$, for both marginally and fully turbulent flow are presented in figure 6. At $R e=1207$ (figure 6a), switching of the flow field between two states is confirmed by the p.d.f. at $y / h=0.3$ being bimodal. The point $y / h=0.3$ has been chosen as it is where the largest difference between the two flow states can be observed in the DNS data of Uhlmann et al. (2007). The data were collected over a period of $12843 h / U_{b}(3600 \mathrm{~s})$, sufficiently long to capture many switches between states. The p.d.f. could be viewed as a combination of two p.d.f.s, one for each flow state; but separating the two is not a trivial task as there is a significant overlap. It can be partially achieved by plotting a p.d.f. of modal velocities (dotted line in figure $6 a$ ). Each modal velocity is taken from a sample of data of the order of $43 h / U_{b}$. The resulting p.d.f. shows the bimodal form created by the two flow states more clearly, although there is still overlap. The values of $U /\langle U\rangle$ at the peaks correspond very well to those extracted from the time averages of Uhlmann et al. (2007) as shown by the dashed vertical lines in figure 6(a).

A bimodal p.d.f. also occurs at $y / h=0.2$ but beyond $y / h=0.4$, this dual-peak feature fades away at higher distances from the wall as the two flow states become increasingly similar (see figure $6 b$ ). In contrast, the p.d.f. for fully turbulent flow at $R e=2234$ and $y / h=0.3$ (figure $6 c$ ) is unimodal, indicating that the flow exists in only 

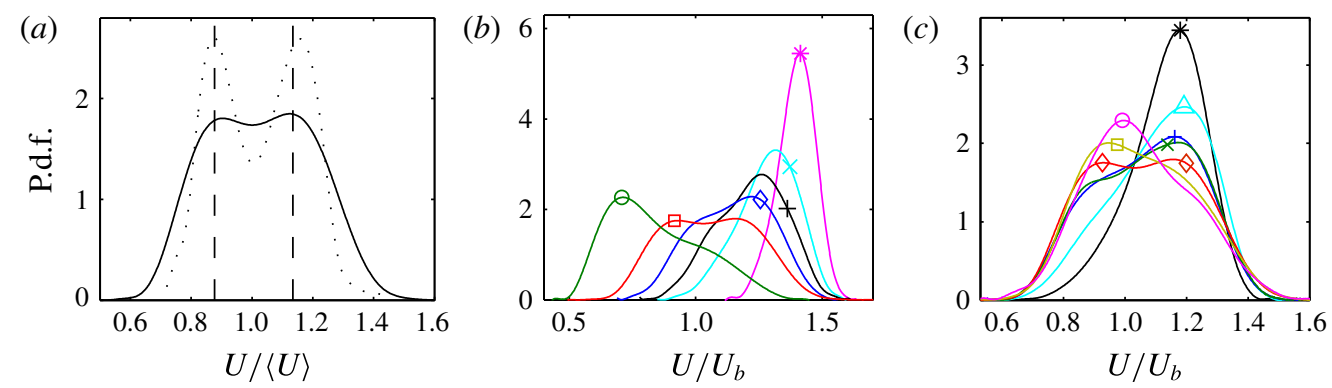

FIgURE 6. (Colour online) Probability density functions of $U / U_{b}:(a) \operatorname{Re}=1207$ and $y / h=0.3$; dotted line is the p.d.f. of modal velocities and dashed lines correspond to short time averages for each state from the DNS data of Uhlmann et al. (2007); (b) $\operatorname{Re}=1203$ : $\bigcirc, y / h=0.2 ; \square, y / h=0.3 ; \diamond, y / h=0.4 ;+, y / h=0.5 ; \times, y / h=0.6 ; *, y / h=1 ;$ (c) $y / h=0.3: \bigcirc, \operatorname{Re}=1097 ; \square, \operatorname{Re}=1125 ; \diamond, \operatorname{Re}=1207 ; \times, \operatorname{Re}=1290 ;+, \operatorname{Re}=1370$; $\triangle, R e=1596 ; *, R e=2234$.

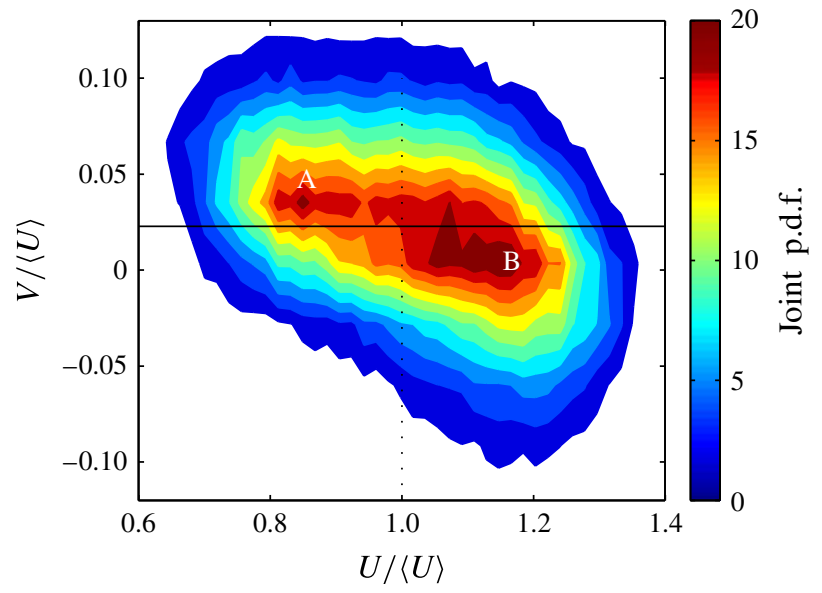

FIgURE 7. (Colour online) Joint p.d.f. at $y / h=0.3$ and $R e=1290$. Data were collected over a period of $7004 h / U_{b}$.

one state. In this study, bimodal p.d.f.s at $y / h=0.3$ were observed between $R e=1097$ and $R e=1370$ as shown in figure 6(c). The two peaks are mostly of different heights, indicating that the flow spends more time in one state than in the other, the largest peak gradually shifting from left to right as the Reynolds number is increased (see figure $6 c$ ).

The joint p.d.f. of streamwise and wall-normal velocities at $R e=1290$ for data collected over a period of $7004 h / U_{b}$ is shown in figure 7. A dual peak can be clearly seen, corresponding to two flow states, A and B. In state A, streamwise velocities lower than the long-term mean (indicated as a dotted line) are highly probable to occur alongside wall-normal velocities which are higher than the long term mean (shown as a solid black line). In state B, streamwise velocities higher than the long-term average have a higher probability of occurring together with essentially zero wallnormal velocities at this measurement location. This behaviour is consistent with the findings of Uhlmann et al. (2007) with regard to the streak-vortex arrangement for 

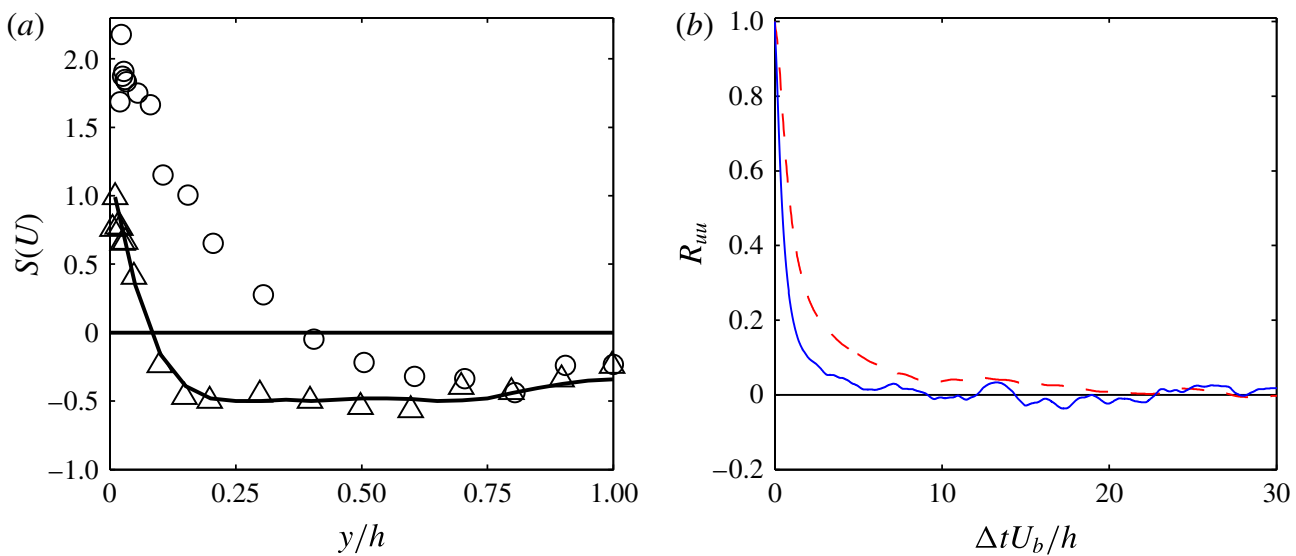

FIgURE 8. (Colour online) (a) Axial velocity skewness along the wall bisector: $\bigcirc$ experiment at $R e=1203 ; \triangle$, experiment at $R e=2230 ;-$, DNS of Gavrilakis (1992) at $R e=2205$. (b) Streamwise velocity autocorrelation at $y / h=0.3$ : $---(\mathrm{red}), R e=1207$; (blue), $R e=2234 ; \Delta t$ is the time lag.

any given pair of opposite walls during marginally turbulent flow. The flow alternating between periods of high turbulence activity, characterised by a low-velocity streak at the wall bisector flanked by vortices (corresponding to state A), and more quiescent periods where there are no streaks and the flow is essentially unidirectional at the measurement location (state B).

Variation of streamwise velocity skewness with distance from the duct wall is shown in figure $8(a)$. The data for $R e=2230$ show excellent agreement with the DNS of Gavrilakis (1992), having positive values very close to the wall and becoming negative beyond $y / h \approx 0.075$. For $R e=1203$, the velocities are more positively skewed in the near-wall region up to $y / h \approx 0.7$, this is due to the presence of the two flow states at the marginally turbulent Reynolds number as shown by the p.d.f.s in figure 6. Beyond $y / h \approx 0.7$, similar values of skewness are observed in both flows.

Streamwise velocity autocorrelation functions $\left(R_{u u}\right)$ for both marginally and fully turbulent flows at $y / h=0.3$ are shown in figure $8(b)$. The marginally turbulent flow is correlated over a larger time period reinforcing the evidence that the flow remains in a particular state for a significant period of time. The integral time scales have been computed by integrating the autocorrelation functions up to the first zero crossing. The integral time scale for marginally turbulent flow $\left(2.24 \mathrm{~h} / U_{b}\right)$ is significantly larger than fully turbulent flow $\left(0.89 h / U_{b}\right)$. Given this evidence for the persistence of each of the two states it is a valid approximation to use Taylor's hypothesis of frozen flow to convert our time-series data into a pseudo-spatial streamwise dimension using an appropriate convection velocity (Taylor 1938; Dennis \& Nickels 2008). It is reasonable to use the bulk velocity as an approximate convection velocity and as such the nondimensional streamwise extent becomes, $\Delta x=\Delta t U_{b} / h$. Therefore figure $8(b)$ can be viewed as a statistical measure of the increased streamwise correlation in marginally turbulent flow. We hypothesise that this is as a result of each of the two states having a significant streamwise distance, and hence there is a spatial switching between the two states along the length of the duct. This is distinct from the temporal switching observed in the axially minimal flow unit in the DNS of Uhlmann et al. (2007). 

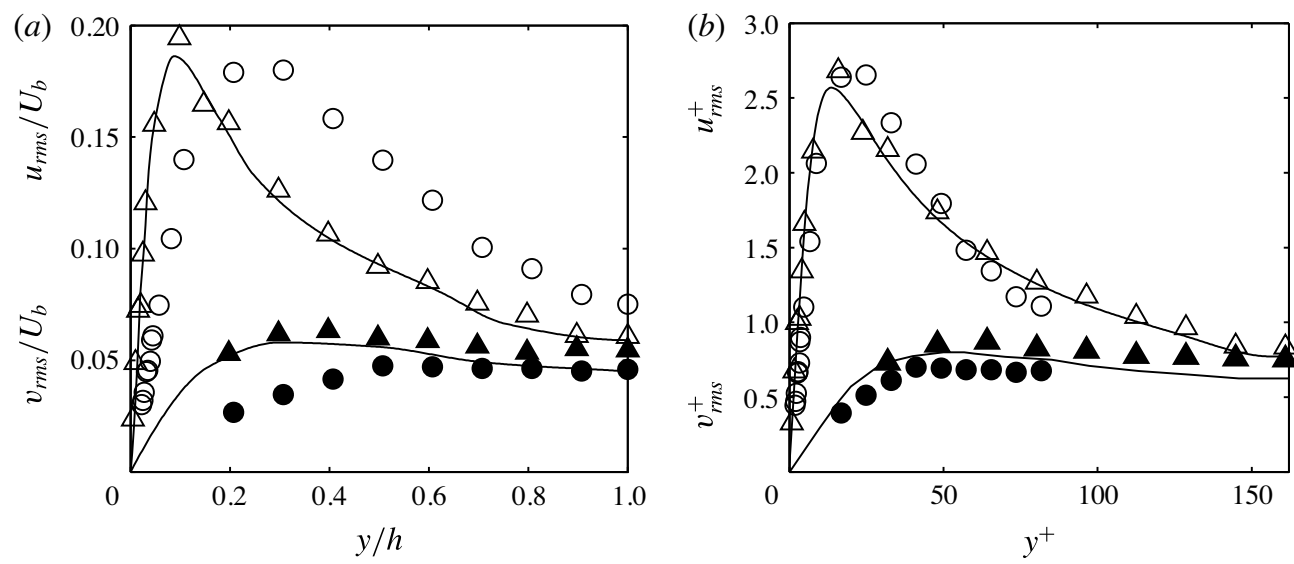

FIGURE 9. Turbulence intensities along the wall bisector: $(a)$ as a function of $y / h ;(b)$ as a function of $y^{+} ; O$, experiment at $R e_{\tau}=81 ; \Delta$, experiment at $R e_{\tau}=161 ;-$, DNS of Gavrilakis (1992) at $R e_{\tau}=162$. Open and closed symbols represent streamwise and wall-normal turbulence intensities, respectively.

\subsection{Turbulence intensity and Reynolds stress measurements}

The streamwise and wall-normal turbulence intensities at $\operatorname{Re}_{\tau}=81(R e=1203)$ and $R e_{\tau}=161(R e=2230)$ as a function of distance from the duct wall along the bisector, in outer units, are shown in figure $9(a)$. It can be observed that $u_{r m s} / U_{b}$ for fully turbulent flow shows good agreement with the DNS of Gavrilakis (1992), attaining a peak at $y / h \approx 0.1$. This peak value is however slightly higher than the DNS result. In the marginally turbulent case, there appears to be a shift in the location of the maximum value of $u_{r m s} / U_{b}$ away from the wall to $y / h \approx 0.3$. Close to the duct wall, the streamwise velocity fluctuations are lower than in fully turbulent flow but become larger as the peak value is approached and remains so across the remaining portion of the duct, creating an impression that the turbulence level is higher. Figure $9(b)$ shows the same data plotted as a function of distance along the bisector in wall units. In this case, the streamwise turbulence intensities, $u_{r m s}^{+}$are very similar in both flows, with $u_{r m s}^{+}$at $R e_{\tau}=81$ becoming lower than the fully turbulent values beyond $y^{+}$of 63 .

The wall-normal turbulence intensities, which are significantly smaller, are almost constant across the duct and no distinct maxima can be identified. The wall-normal turbulence intensities at $R e_{\tau}=161$ show good agreement with DNS data, they are however a little higher than the simulation values towards the duct centre and larger than those at $R e_{\tau}=81$. The results indicate that near the centre of the duct, the turbulence is nearly isotropic, as shown by the closeness of $u_{r m s}^{+}$and $v_{r m s}^{+}$values at high $y^{+}$.

Figure 10 shows the variation of Reynolds shear stress along the wall bisector. The data have been normalised by the square of the bulk velocity. Since only the value of local friction velocity $\left(u_{\tau}\right)$ at the wall midpoint is available in this experiment, normalising by $u_{\tau}^{2}$ will not provide a true picture of Reynolds shear stress distribution. The data for $R e=2230$ is in good agreement with DNS. At $R e=1203$, Reynolds shear stress can be observed to be lower for $y / h<0.4$. There is also a shift in location of the maximum away from the duct wall. As the duct centre is approached, Reynolds shear stress drops to zero for both flows as it must due to symmetry. 


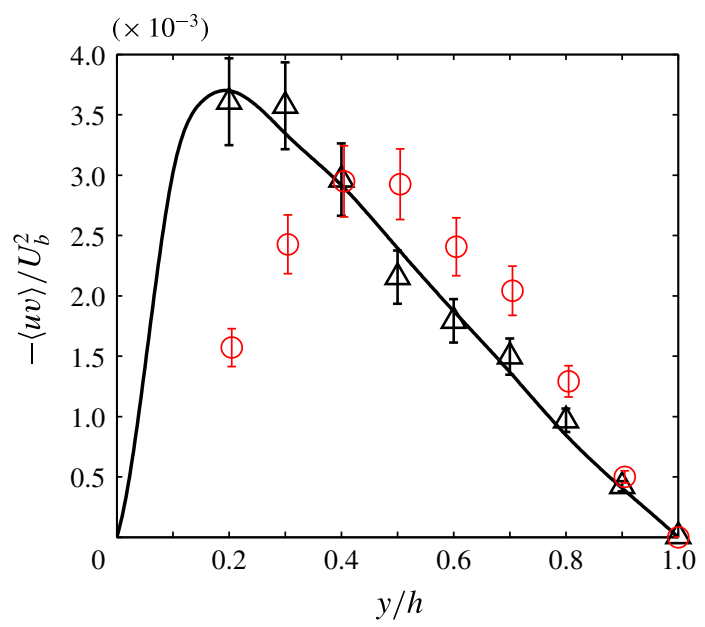

FIGURE 10. (Colour online) Variation of Reynolds shear stress along the wall bisector: $\bigcirc$ (red) experiment at $R e=1203 ; \Delta$, experiment at $R e=2230$;, DNS of Gavrilakis (1992) at $R e=2205$.

\section{Conclusion}

The behaviour of turbulent flow in a square duct at relatively low Reynolds numbers has been studied. The results for both marginally turbulent flow at $R e_{\tau}=81$ and fully turbulent flow at $R e_{\tau}=161$ show good agreement with the DNS data of Uhlmann et al. (2007) and Gavrilakis (1992), respectively. It has been shown that the onset of turbulence can be significantly affected by Coriolis effects due to the Earth's rotation. This is as a result of differences in the laminar base flow velocity profiles at different values of Ekman number prior to transition. A limiting Reynolds number of about 940 for transition was observed at $E k \approx 7$. This value is within the range obtained in previous numerical studies.

In marginally turbulent flow a mean flow very similar to laminar flow has been observed in the vicinity of the duct wall, as well as a large overshoot from the logarithmic law nearer the centre. Switching between two flow states, originally predicted by the DNS of Uhlmann et al. (2007), is confirmed by bimodal p.d.f.s of streamwise velocity at certain distances from the duct wall and a joint p.d.f. of streamwise and wall-normal velocity which features two peaks corresponding to each of the two states: one essentially unidirectional $\left(v^{+} \approx 0\right)$ at the measurement location and the other containing a significant secondary flow component $(V /\langle U\rangle \approx 0.03)$.

It has been shown that marginally turbulent flow is more correlated than a fully turbulent flow as indicated by its longer integral time scale. Similar levels of $u_{r m s}^{+}$have been observed in both flows at different $y^{+}$. The Reynolds stresses in the near-wall region are however lower in the former but become very similar to those of fully turbulent flow as the duct centre is approached.

\section{Acknowledgements}

The authors wish to thank Professor Uhlmann of the Institute of Hydromechanics, University of Karlsruhe, Germany for providing data from their DNS for comparison with our experiments. R.J.P. acknowledges funding for a 'Fellowship in Complex Fluids and Rheology' from the Engineering and Physical Sciences Research Council (EPSRC, UK) under grant number EP/M025187/1. 


\section{REFERENCES}

Biau, D. \& Bottaro, A. 2009 An optimal path to transition in a duct. Phil. Trans. R. Soc. Lond. A 367 (1888), 529-544.

BRUndRETT, E. \& BAINES, W. D. 1964 The production and diffusion of vorticity in duct flow. J. Fluid Mech. 19 (03), 375-394.

Dennis, D. J. C. \& Nickels, T. B. 2008 On the limitations of Taylor's hypothesis in constructing long structures in a turbulent boundary layer. J. Fluid Mech. 614, 197-206.

Draad, A. A. \& Nieuwstadt, F. T. M. 1998 The Earth's rotation and laminar pipe flow. J. Fluid Mech. 361, 297-308.

Escudier, M. P., Poole, R. J., Presti, F., Dales, C., Nouar, C., Desaubry, C., Graham, L. \& Pullum, L. 2005 Observations of asymmetrical flow behaviour in transitional pipe flow of yield-stress and other shear-thinning liquids. J. Non-Newtonian Fluid Mech. 127 (2), $143-155$.

EsCUdieR, M. P. \& SMith, S. 2001 Fully developed turbulent flow of non-Newtonian liquids through a square duct. Proc. R. Soc. Lond. A 457 (2008), 911-936.

GAVRILAKIS, S. 1992 Numerical simulation of low-Reynolds-number turbulent flow through a straight square duct. J. Fluid Mech. 244, 101-129.

HoAgland, L. C. 1960 Fully developed turbulent flow in straight rectangular ducts: secondary flow, its cause and effect on the primary flow. PhD thesis, Massachusetts Institute of Technology, Cambridge.

Huser, A. \& Biringen, S. 1993 Direct numerical simulation of turbulent flow in a square duct. J. Fluid Mech. 257, 65-95.

Hutchins, N.\& CHOI, K. S. 2002 Accurate measurements of local skin friction coefficient using hot-wire anemometry. Prog. Aerosp. Sci. 38 (4), 421-446.

Joung, Y., ChOI, S. \& CHOI, J. 2007 Direct numerical simulation of turbulent flow in a square duct: analysis of secondary flows. J. Engng Mech. ASCE 133 (2), 213-221.

LAUnder, B. E. \& YING, W. M. 1972 Secondary flows in ducts of square cross-section. J. Fluid Mech. 54 (02), 289-295.

Melling, A. \& Whitelaw, J. H. 1976 Turbulent flow in a rectangular duct. J. Fluid Mech. 78 (02), 289-315.

NIKURADSE, J. 1926 Untersuchungen über die geschwindigkeitsverteilung in turbulenten strömungen. PhD Thesis, Gottingen; VDI Forsch p. 281.

OKino, S., NAGATA, M., Wedin, H. \& BotTARo, A. 2010 A new nonlinear vortex state in squareduct flow. J. Fluid Mech. 657, 413-429.

Pinelli, A., Uhlmann, M., Sekimoto, A. \& Kawahara, G. 2010 Reynolds number dependence of mean flow structure in square duct turbulence. J. Fluid Mech. 644, 107-122.

Sekimoto, A., Pinelli, A., Uhlmann, M. \& Kawahara, G. 2009 The effect of coherent structures on the secondary flow in a square duct. In Advances in Turbulence XII (ed. B. Eckhardt), Springer Proceedings in Physics, vol. 132, pp. 329-332. Springer.

TAtsumi, T. \& Yoshimura, T. 1990 Stability of the laminar flow in a rectangular duct. J. Fluid Mech. 212, 437-449.

TAYlor, G. I. 1938 The spectrum of turbulence. Proc. R. Soc. Lond. A 164 (919), 476-490.

Uhlmann, M., Kawahara, G. \& Pinelli, A. 2010 Traveling-waves consistent with turbulencedriven secondary flow in a square duct. Phys. Fluids 22 (8), 084102.

Uhlmann, M., Pinelli, A., Kawahara, G. \& Sekimoto, A. 2007 Marginally turbulent flow in a square duct. J. Fluid Mech. 588, 153-162.

Wedin, H., Bottaro, A. \& Nagata, M. 2009 Three-dimensional traveling waves in a square duct. Phys. Rev. E 79, 065305.

White, F. M. 2006 Viscous Fluid Flow, 3rd edn. McGraw-Hill. 